\title{
ADULTOS MAYORES FUNCIONALES: UN NUEVO CONCEPTO EN SALUD
}

\author{
FUNCTIONALSELDERS: A NEW CONCEPT IN HEALTH \\ MARCELA SANHUEZA PARRA*, MANUEL CASTRO SALAS** \\ Y JOSÉ M. MERINO ESCOBAR***
}

\begin{abstract}
RESUMEN
Se revisa el concepto de funcionalidad en el adulto mayor, dentro de un contexto internacional y nacional, mencionando investigaciones realizadas en nuestro país y generalidades en relación a instrumentos de evaluación más utilizados, junto al papel del sector salud y en especial de enfermería. Finalmente se propone un programa de actividades destinadas a aumentar la funcionalidad del adulto mayor.
\end{abstract}

Palabras claves: Adulto mayor, funcionalidad, salud, valoración geriátrica.

\begin{abstract}
ABST RACT
The article reviews the concept of functionality in the elders, within an international and national context, mentioning researches carried out in our country and some general facts in relation to the most used instruments of evaluation, in addition to the function of the health field and especially of thenursing field. Finally, it proposes an activity program devoted to increase the elder's functionality.
\end{abstract}

Keywords: Elder, functionality, health, geriatric assessment.

Fecha recepción: 29/06/04. Fecha aceptación: 19/08/05.

\section{INTRODUCCIÓN}

Latransición demográficaqueviveChiley que setraduce, en términossimples, en un aumento importantedela población adulta mayor, conlleva cambios profundos y significativos en la convivencia social y enfoque sanitario (Instituto Nacional de Estadística, 1999).

El interés en el senescentees mayor, dejando atrás conductas del "modelo médico tradicional", donde la actitud frente al anciano se ve desfavorecida, presentándolo como sinónimo de decadencia, déficit einvolución (Fornós, 1996).
La Organización Panamericana de la Salud (OPS) define el estado de salud entre los envejecidos no en términos de déficit sino de mantenimiento de la capacidad funcional (OPS/OMS, 1982).

El presente artículo tiene como objetivo entregar una descripción del concepto funcionalidad en el adulto mayor, dentro de un contexto internacional y nacional, finalizando con una propuesta deactividades enmarcadas dentro de un programa que elevaría la funcionalidad de los envejecidos.

\footnotetext{
* M arcela I sabel del Pilar Sanhueza Parra. Enfermera, Licenciada en Enfermería, M agíster en Enfermería Universidad de Concepción. Dirección: Versalles 2631 A.A del Canto, Talcahuano, Chile. Fono: (56-41) 434542. E-mail: sanhuezaparra @gmail.com

** M anuel Castro Salas. Enfermero, Sociólogo, Magister en Enfermería mención Salud Comunitaria. Profesor Asociado Universidad deConcepción. E-mail: mcastro@udec.cl

*** Josè M anuel Merino Escobar. Profesor Titular. Sociólogo, Magíster en Ciencias Sociales, PhD in Sociology. Universidad de Concepción. Direcciòn: Roosevelt s/n, Concepción-Chile. Teléfonos: (56-41) 204826 - (56-41) 207064. Fax: (56-41) 228353. E-mail: jmerino@udec.cl
} 


\section{CONTEXTO INTERNACIONAL}

La Segunda Asamblea Mundial sobre el Envejecimiento del año 2002, celebrada en $\mathrm{M}$ adrid (OPS/OM S, 2002), convocó a 142 países miembros de las Naciones Unidas a objeto de evaluar el estado de avance de las recomendaciones del Plan deAcción de Envejecimiento dela Primera Asamblea celebrada en Viena en 1982 (Naciones Unidas, 1982) y aprobar una declaración política delos gobiernosy un plan deacción internacional quemejoraría las condiciones de vida de la población adulta mayor.

En general, las políticas internacionales van orientadas a un cambio en la concepción de la vejez, considerando a ésta como una etapa de vida activa, en la que se puede lograr el máximo deautonomía individual y la posibilidad dela autorrealización (Belenguer, 2000).

Así, la planificación y distribución de los servicios para los ancianos debe dar respuesta a sus necesidades específicas, e idealmente el sistema de servicios debe incluir "servicios para el anciano relativamente sano e independiente, servicios para aquellos que experimentan limitaciones y requieren apoyos, servicios para aquellos cuyas necesidades requieren cuidado institucionalizado" (OPS, 1997).

\section{CONTEXTO NACIONAL}

\subsection{Antecedentes demográficos}

Demográficamente, al igual queel resto de los países de Latinoamérica, Chile presenta un cambio progresivo y acelerado de su pirámide poblacional; es el tercer país más envejecido de Latinoamérica, donde el grupo de 60 años o más -que en el año 2001 era de un $10,6 \%$ - se incrementará a un $19 \%$ en el año 2025 , con una expectativa de vida que alcanzará los 80 años (M inisterio de Planificación, 2003).

\subsection{Políticas y programas}

Si se analiza la trayectoria histórica del país en relación a la preocupación por los problemas y demandas de los ancianos, ésta es relativamente nueva, comienza en los años 80, cuando seincluyeen las agendas dediscusión política aquellos aspectos relativos al envejecimiento y sus consecuencias (OPS, 1994).

Dentro de la "Política Nacional del Adulto M ayor", en el año 1996 (ComitéN acional del Adulto M ayor, 1996) se plantea como objetivo principal "lograr un cambio cultural que dé un mejor trato a la población adulta mayor", lo que implica una percepción distinta del envejecimiento con la consiguiente necesidad de crear medios para facilitar el desarrollo integral del adulto mayor y lograr el mantenimiento o recuperación de la funcionalidad de este grupo etáreo.

Por su parte, el sector salud desarrolla la "Política de Salud del Adulto Mayor", cuyos objetivos principales están dirigidos al mantenimiento de la funcionalidad y reducción de la mortalidad por causas evitables.

Los programas que hoy aparecen como imprescindibles para el apoyo integral del anciano son en su mayoría aquellos orientados a la salud y los servicios sociales.

En nuestro paísel M inisterio deSalud (M IN $\mathrm{SAL}$ ) cuenta con el programa del adulto mayor donde se tratan, previenen y rehabilitan diversas patologías, tanto de adultos mayores autovalentes, frágiles y postrados ( $M$ inisterio de Salud, 2002).

\subsection{Barreras naturales}

Aunque la preocupación del país en favor del adulto mayor se ha incrementado, existen situaciones naturales queacompañan al senescente y pueden crear barreras a los propósitos planteados; entre éstas: la alta prevalencia de las enfermedades crónicas, secuelas invali- 
dantes y cambios físicos que acompañan el proceso deenvejecimiento, adicionando el efecto dela llamada "profecíaautocumplida", lacual convenceal anciano quesu condición es sinónimo de deterioro y carga social (Salvarezza, 1999).

\section{EL PAPEL DE LA FUNCIONALIDAD}

Desde una perspectiva funcional, un adulto mayor sano es aquel capaz de enfrentar el proceso de cambio a un nivel adecuado de adaptabilidad funcional y satisfacción personal (OM S, 1985). De esta forma, el concepto de funcionalidad esclave dentro dela definición de salud para el anciano, por ello la OMS (1982) proponecomo el indicador más representativo para este grupo etáreo el estado de independencia funcional.

Fillenbaum (1984) confirma este criterio, sugiriendo la evaluación de salud delos adultos mayores en términos de estatus funcional, con el concepto desal ud asociado al mantenimiento de la independencia funcional.

La funcionalidad o independencia funcional es la capacidad de cumplir acciones requeridas en el diario vivir, para mantener el cuerpo y subsistir independientemente, cuando el cuerpo y la mente son capaces de llevar a acabo las actividades de la vida cotidiana se dicequelafuncionalidad estáindemne(M IN SAL, 2003).

\section{INSTRUMENTOS DE EVALUACIÓN}

A principios de los sesenta se crean los primerosinstrumentos parala medición de funcionalidad, como las escalas de Kast y Barthel, los que han sido corregidos y reformulados a través del tiempo. Como ejemplo están: la escala de la Cruz Roja españolay el OIder Multidimensional Resourses and Services (OARS), entreotros (Perlado, 1995), losquea su vez se acompañan de instrumentos que evalúan el deterioro cognitivo o las funciones mentales, indicadores que influyen directamente sobre la funcionalidad (Pedraza, 1992b).

\section{FUNCIONALIDAD EN CHILE}

La investigación sobrefuncionalidad en Chile no ha sido un tema de gran trayectoria investigativa. En el año 1985 el sociólogo Oscar Domínguez midió - como parte de un estudio internacional apoyado por la OPS- el nivel deautonomía en la población chilena, que reveló las siguientes categorías y porcentajes: autónomos 0 autovalentes, 66,7\%; frágiles $30 \%$ y totalmente dependientes, 3,3\%. A su vez, sedescribieron las características generales de tipo socioeconómico para cada grupo (Domínguez,1987).

Posteriormente, en el año 2002, el Sistema Nacional de Servicios de Salud (SNSS) realizó una preevaluación de la funcionalidad de adultos mayores atendidos en el nivel primario, se utilizó la escala modificada de la Cruz Roja española, y el propósito fue evaluar la funcionalidad, para posteriormenteaplicar un instrumento que reuniera las condiciones para medir con eficiencia lafuncionalidad del adulto mayor.

Actualmenteel instrumento existey seaplica, secreó el año 2003 y lleva por nombre Evaluación Funcional del Adulto M ayor (EFAM).

\section{APORTE DEL PERSONAL SANITARIO}

En el sector salud se produce un verdadero cambio de paradigma. Se pasa desde un modelo que dio prioridad a la salud maternoinfantil centrada en superar principalmente las enfermedades infecciosas en la década del 60 , a un modelo que pone énfasis en la atención primaria, siendo uno de sus principales objetivos la atención de losadultos mayoresa fines de la década del 80 (Vio, 2000).

Seestáfrentea un grupo que requiereaten- 
ción y cuidados que superen las limitaciones del paradigma biomédico. Es así como el propósito delos cuidados en salud, en particular para los profesionales de enfermería -quienes tienen especial orientación hacia el cuidado-, será evitar que estas limitaciones se conviertan en impedimentos para el desarroIlo deactividades queel adulto mayor necesite o desee realizar, y se deberán esforzar en disminuir el nivel de dependencia deeste grupo etáreo y las oportunidades en que estas personas precisen ayuda asistencial.

\section{MEDIDAS DE ABORDAJE}

Para el logro del mantenimiento de la funcionalidad, el M inisterio de Salud hainiciado programas de capacitación queseencuentran en una etapa de sensibilización masiva de los diferentes niveles asistenciales y equipos de salud. A su vez, si deseamos aumentar los niveles de funcionalidad de nuestros ancianos, debemos detectar en forma anticipada aque llas situaciones presentes en el adulto mayor que coloquen en riesgo la mantención de ella (M arín, 1998), lo que debe ser realizado por los equipos del nivel primario deatención, por lo cual la autoridad de salud debe preocuparse de sensibilizar y mantener programas de actualización destinadosa quienes trabajan en la atención primaria.

Como una forma decontribuir al desarrollo de la funcionalidad y autonomía de la población adulta mayor, se propone la realización de un programa integral de actividades orientadas a satisfacer esta necesidad, el propósito será potenciar la Autonomía Funcional de los Adultos Mayores, lo que se podrá lograr a través de los siguientes objetivos esperados para los adultos mayores que participen de éste programa:

- Valorar la importancia de participar en un programa destinado a estimular la autonomía funcional.
- Identificar el autocuidado como una estrategia que conduce a estilos de vida saludables. - Propiciar la interacción espontánea y cordial, junto con estimular la amistad y colaboración entretodos.

Los temas que permitirían dar cumplimiento a los objetivos mencionados serían:

$\mathrm{N}$ 1 "Autonomía funcional yadulto mayor". $\mathrm{N}$ - 2 "Autocuidado y salud".

$\mathrm{N}$ ㅇ 3 "Relajación y adulto mayor" № 4 "Recreación: Uso del tiempo libre". $\mathrm{N}$ ㄴ 5 "La comunicación en el adulto mayor".

\section{COMENTARIO FINAL}

Los cambios naturales y problemas de salud que experimenta el adulto mayor setraducen en la declinación de sus capacidades funcionales, las que alcanzan su mayor expresión en el adulto joven, y en la etapa deenvejecimiento disminuyen notoriamente (Forciea, 1996).

Si se desea aumentar los niveles de funcionalidad del anciano, se debe detectar en forma anticipada aquellas situaciones presentes en el adulto mayor que coloquen en riesgo la mantención de ella, con la ayuda de instrumentos creados específicamente para este fin (Pedraza, 1992a).

Estasmedidas deben ser llevadas a cabo por los equipos del nivel primario deatención, es por esta razón quela autoridad de salud debe preocuparse de sensibilizar y mantener programas deactualización destinadosa quienes trabajan en este nivel, que es el lugar más indicado para tratar tempranamentelas dificultades que aquejan a este grupo etáreo.

El ser humano, como ser social, en todas las etapas de su vida está intercambiando experiencias y conocimientos con su medio, por tanto el aprendizaje no es algo exclusivo de losgruposjóvenes dela población (OPS, 1999). Los adultos mayores de igual forma pueden continuar en esteproceso deaprendizajecomo 
educandos, pero la perspectiva desde la cual se les eduque debe ser diferente, esto es, adecuarse a las características personales por las que está atravesando en esta etapa de su vida (Carstensen, 1990).

Los programas educativos destinados a potenciar la funcionalidad entre los ancianos deberían contener actividades queles ayuden a fortalecer aquellas potencialidades que han permanecido en desuso (M arin, 1993), entre ellas podemos mencionar la capacidad de crear, relacionarse, comunicar, aprender, autorrealizarse, dar y recibir afecto, preocupación por otros, cultivar la espiritualidad y encontrar el sentido de la vida en esta etapa de su existencia (Selby, 1986).

\section{REFERENCIAS BIBLIOGRÁFICAS}

Belenger, M .V. y Aliaga, F. (2000). Autonomía funcional y ocupación del tiempo libreen personas mayores. Rev. Rol de Enfermería. España; 23(3):231-234.

Carstensen, L. y Edelstein, B. (comp.) (1990). Gerontología clínica: Intervención psicológica y social. Barcelona, España: Editorial Martínez Roca S.A.

Comité Nacional del Adulto Mayor (1996). Política nacional para el adulto mayor. Documento completo. Chile.

Domínguez, O. (1987). Estudio de las necesidades de la población adulta mayor de 60 años y más en Chile. Simposio Internacional sobre envejecimiento. Chile: Instituto Latinoamericano y del Caribe de Planificación Económica y Social.

Fillenbaum, G. (1984). The wellbeing of the elderly, Approachesto multidimensional assessment, WHO, Offset Publications, $\mathrm{N}^{\circ} 84$.

Forciea, M . y Lavizzo-M ourey, R. (1996). Secretos de la geriatría. M éxico: Ed. M cGraw-Hill Interamericana.

Fornós, M . (1996). Estereotipos, prejuicios y vejez, Rev. Gerontológica La Tardor. España (3): 9-12.

Instituto Nacional deEstadística (INE) (1999). Chiley los adultos mayores, impacto en la sociedad del 2000. Chile.

Langarica, R. (1985). Gerontología y geriatría. M éxico: Ed. Nueva Interamericana, primera ed.

Marín, P. (1998). La situación del adulto mayor en Chile. Rev. M éd Chile; 125: 1207-12.

--------. (1993). Tiempo nuevo para el adulto mayor. Enfoque interdisciplinario. Programa para el
Adulto M ayor VicerrectoríaAcadémica. Chile: Pontificia Universidad Católica de Chile, segunda ed.

Ministerio de Planificación (2003). Programa Chile Solidario, Proyecto Adultos mayores de 65 años que viven solos. Santiago de Chile.

Ministerio de Salud/Instituto Nacional de Geriatría (2003). Evaluación funcional deadulto mayor. Proyecto FON DEF, Santiago de Chile.

Ministerio de Salud (2002). O rientaciones de la atención integral en salud del adulto mayor. Chile. 61 pp.

Naciones U nidas (1982). Plan deAcción Internacional de Viena sobre el Envejecimiento, Asamblea sobre el Envejecimiento, Viena-Austria, julio-agosto.

Organización Panamericana de la Salud, OPS/OMS (2002). Boletín Segunda Conferencia Internacional sobre el Envejecimiento. M adrid, España.

Organización Panamericana de la Salud, OPS/OMS (1999). Sigamos activos para envejecer bien. Día Mundial de la Salud. Boletín oficial, 7 de abril. Washington DC, EE.UU.

Organización Panamericana de la Salud (1997). Boletín Día Mundial de la Salud, Washington DC, EE.UU.

Organización Panamericana de La Salud (1994). Organización M undial dela Salud. La atención delos ancianos: Un desafío para los años noventa. Washington, DC; EE.UU.

Organización Panamericana de La Salud (1993). Enfermería gerontológica: Conceptos para la práctica. Serie Paltex No 31; Washington DC, EE.UU.

Organización M undial de la Salud, OPS/OM S (1985). Hacia el bienestar delos ancianos. Washington DC, EE.UU.

Organización Panamericana de la Salud, OPS/OMS (1982). Evaluación de la situación y adopciones de políticas para la atención de la población de edad avanzada. Washington DC; EE.UU. Documento oficial № 179.

Pedraza, M . y Germán, C. (1992) . Autonomía funcional en ancianos. Rev. Rol de Enfermería España, 15(172):15-9.

(1992). E.V.A. Escala de VaIoración delaAutonomía para el Autocuidado. Rev. Index de Enfermería. España; 1(2): 35-38.

Perlado, F. (1995). Teoría y práctica de la geriatría. M adrid-España: Ed. Díaz de Santos.

Salvarezza, L. (1999). La vejez. Argentina: Ed. Paidós.

Selby, P. y Griffiths, A. (1986). Guía para un envejecimiento satisfactorio. Inglaterra: Ed. Parthenon Publishing, primera ed.

Vio, F. y Albala, C. 2000. Nutrition policy in theChilean Transition. Public Health Nutrition; 3: 49-55. 